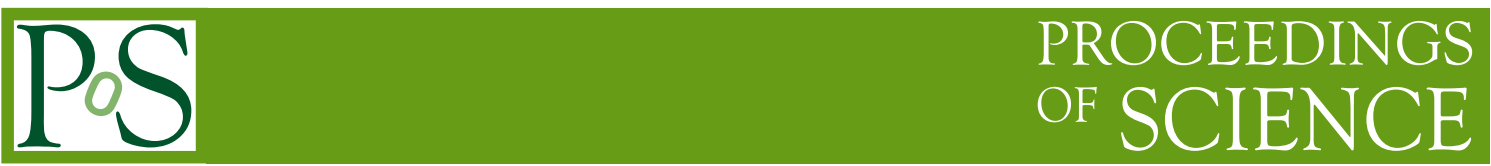

\title{
Charm and Beauty production at the TeVatron
}

\author{
Tara Shears ${ }^{* \dagger}$ \\ University of Liverpool, UK \\ E-mail: tara@hep.ph.liv.ac.uk
}

\begin{abstract}
Measurements of charm and beauty production at the Tevatron have historically shown hints of disagreement when compared to Next to Leading Order predictions. In this review four measurements performed with recent $p \bar{p}$ collision data at $\sqrt{s}=1.96 \mathrm{TeV}$ by the CDF and D0 collaborations are presented: inclusive muon tagged jet production; inclusive beauty (b) jet production; the production of b $\bar{b}$ jets; the production of beauty and charm jets in association with a high energy photon. In all cases measurements are in agreement with theoretical prediction.
\end{abstract}

International Europhysics Conference on High Energy Physics

July 21st - 27th 2005

Lisboa, Portugal

\footnotetext{
*Speaker.

$\dagger$ On behalf of the CDF and D0 Collaborations.
} 


\section{Introduction}

Previous measurements of charm (c) and beauty (b) quark production at the Tevatron have shown discrepancies when compared to Next to Leading Order (NLO) predictions [1]. Improved predictions have reduced this discrepancy [2] although large theoretical uncertainties still exist.

For this reason it is vital that both the CDF and D0 experiments perform updated measurements of beauty and charm production. In this review four measurements sensitive to heavy quark production are presented: inclusive muon tagged jet production; inclusive $b$ jet production; the production of $b \bar{b}$ jets; the production of $b$ and $c$ jets in association with an energetic photon. More information concerning other measurements of heavy quark production can be found elsewhere [3].

\section{Inclusive muon tagged jet production}

The D0 Collaboration has measured the production of jets containing muons in $294 \mathrm{pb}^{-1}$ of data. This topology contains contributions from the semi-leptonic decays of both bottom and charm quarks. Events are selected by requiring that there be at least one jet within a pseudorapidity $(\eta)$ region of $|\eta|<0.5$ which contains a muon with transverse momentum $\left(p_{T}\right)$ exceeding $5 \mathrm{GeV}$. Jets of transverse energy $\left(E_{T}\right)$ ranging between 90 and $400 \mathrm{GeV}$ are considered in the analysis. The heavy quark component of the selected events is estimated from Pythia Monte Carlo (leading order matrix element + parton shower), and the production cross-section of heavy flavour jets containing muons extracted. This is compared to Pythia and NLO (NLOJET++ [4] multiplied by the heavy flavour fraction found in Pythia) predictions in figure 1. It can be seen that the data lie between Pythia and NLO predictions, and are consistent with both once the systematic error is taken into account.

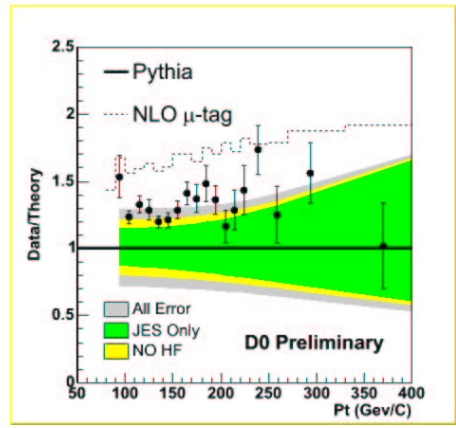

Figure 1: Ratio of inclusive muon tagged jet cross-section in data (points) to Pythia prediction, shown as a function of jet $p_{T}$. An estimated $\mathrm{k}$-factor between NLO and LO predictions is shown by the dashed line. The shaded band represents the systematic error on the ratio, which is also broken down into jet energy scale (JES) and heavy flavour fraction (HF) components.

\section{Inclusive b jet production}

The CDF Collaboration has measured inclusive $\mathrm{b}$ jet production in $300 \mathrm{pb}^{-1}$ of data. Events are selected by requiring a jet within $|\eta|<0.7$ which contains a separated secondary vertex. 

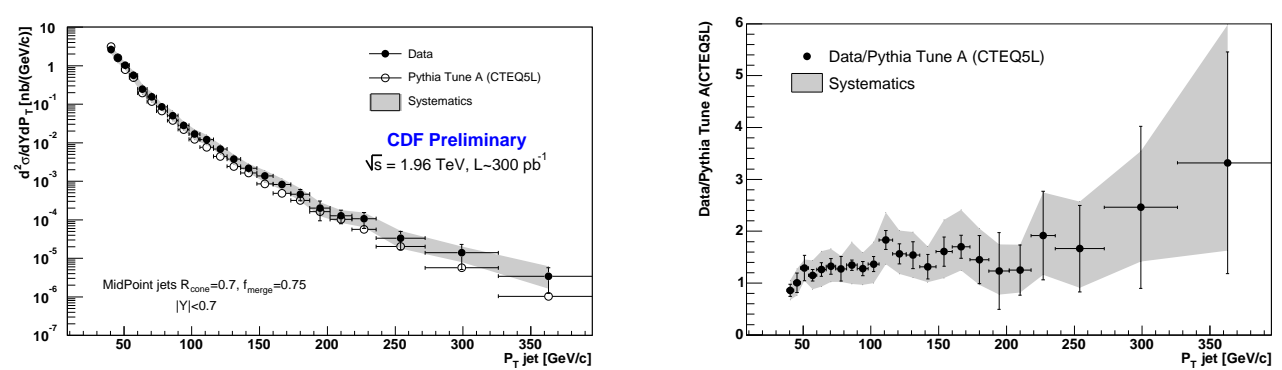

Figure 2: (Left)Inclusive b jet cross-section, (right) ratio of cross-section measured in data to that predicted in Pythia, both shown as a function of jet $p_{T}$. In both cases data are shown by points, and systematic error on the measurement by the shaded band. The open points on the left show Pythia predictions.
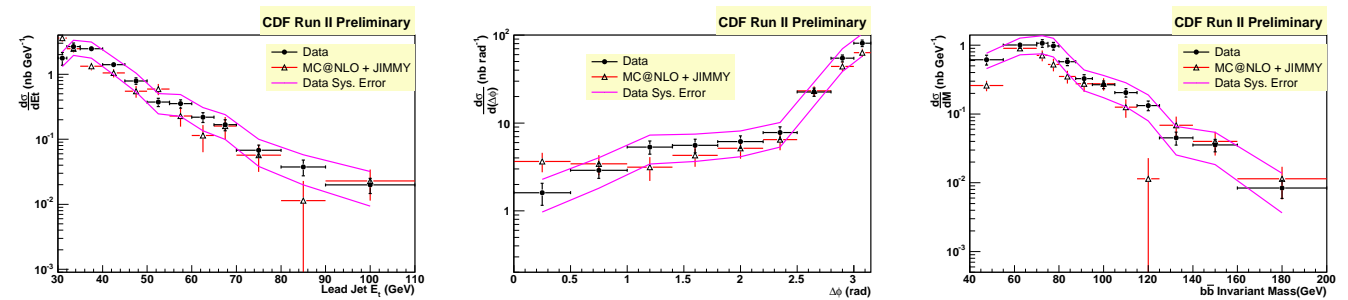

Figure 3: Differential b $\bar{b}$ dijet production cross-sections shown as functions of (left) leading jet $E_{T}$; (centre) azimuthal angle between jets; (right) dijet invariant mass. In all cases data are denoted by the black points, NLO predictions by the open points, and the systematic error on the measurement denoted by the outlined band.

The fraction of events containing $b$ jets is estimated by fitting the invariant mass of the secondary vertex to template shapes expected for $\mathrm{b}$ and other jets. Figure 2 shows the cross-section for $\mathrm{b}$ jet production compared to Pythia prediction. The figure also shows the ratio of data to Pythia predictions as a function of jet $p_{T}$. The data lie consistently above Pythia predictions, but are consistent within the systematic error. NLO comparisons are expected in the near future.

\section{4. $b \bar{b}$ dijet production}

It is also possible to test predictions of heavy quark production by isolating specific production mechanisms in data and comparing those to theoretical prediction. The CDF Collaboration has measured the production cross-section of $b \bar{b}$ dijets in a small sample of data. Events are selected by requiring two jets within $|\eta|<1.2$ which both contain a separated secondary vertex. This is a topology which is enriched in LO production mechanisms. The $E_{T}$ of the most (least) energetic jet must exceed 30 (20) GeV. The fraction of events arising from b quarks is estimated from fitting the secondary vertex mass distribution in data to templates for $\mathrm{b}, \mathrm{c}$ and light quarks. The production cross-section is then estimated as a function of most energetic (leading) jet $E_{T}$, invariant mass of

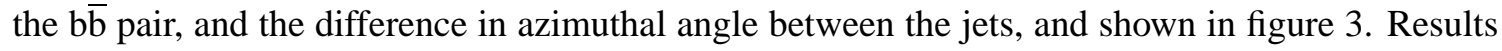
have been compared to Pythia (not shown here) and MC@ NLO predictions [5] which include an (untuned) estimate of the underlying event [6]. In all cases data are consistent with NLO prediction. 

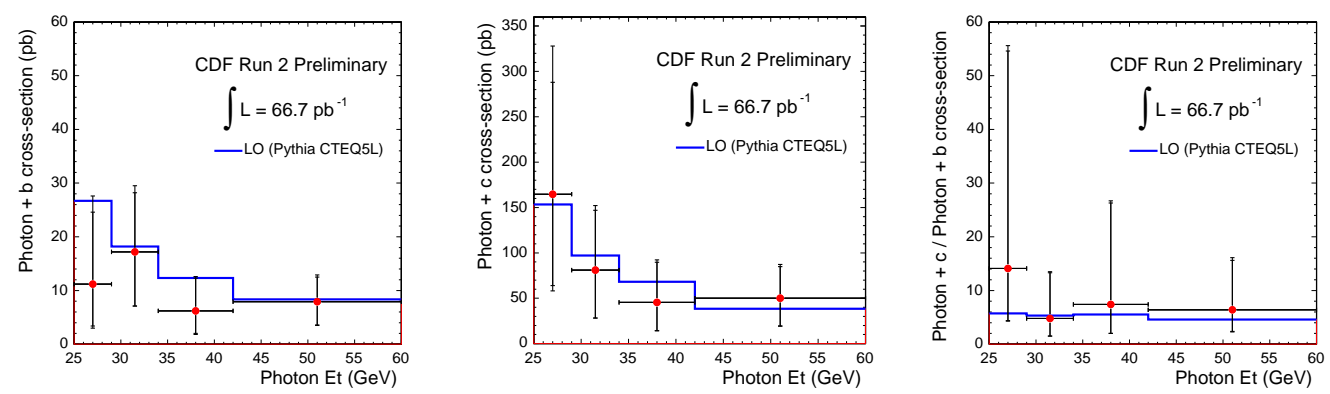

Figure 4: Cross-sections for photon $+b$ (left), photon $+c$ (centre), and ratio of photon $+c$ to photon $+b$ cross-sections (right), shown as a function of photon $E_{T}$. In all cases the points represent data, the error bars show statistical and systematic errors on the measurement, and the line shows Pythia predictions.

\section{5. $b$ and $\mathbf{c}$ jet production in association with a photon}

One can also test different heavy quark production mechanisms by measuring heavy quark production in association with a gauge boson. These topologies are often enriched in NLO processes such as gluon splitting. The CDF Collaboration has measured the cross-section of $\mathrm{b}$ and $\mathrm{c}$ quarks produced in association with a photon in $67 \mathrm{pb}^{-1}$ of data. Events are selected by requiring a photon within $|\eta|<1.0$ with $E_{T}>25 \mathrm{GeV}$, and a jet containing a separated secondary vertex. The fraction of events containing $\mathrm{b}$ and $\mathrm{c}$ quarks is estimated by fitting the invariant mass of the secondary vertex as before. After photon backgrounds are subtracted the cross-section for photon $+\mathrm{b}$ and photon $+\mathrm{c}$ production is calculated as a function of photon $E_{T}$ and shown in figure $\emptyset$ compared to Pythia predictions. The cross-section ratio is also shown. It can be seen that within the limited statistics available data are consistent with Pythia predictions.

\section{Conclusions}

Measurements of heavy quark production at the CDF and D0 experiments have been presented. All measurements are in agreement with theoretical predictions.

\section{References}

[1] See, for example: D. Acosta et al. Phys. Rev. D65 052005 (2002);

F. Abe et al.: Phys. Rev. Lett. 751451 (1995), Phys. Rev. Lett. 71500 (1993), Phys. Rev. Lett. 693704 (1992);

B. Abbott et al.: Phys. Lett. B487 264 (2000), Phys. Rev. Lett. 845478 (2000), Phys. Rev. Lett. 85 5068 (2000);

S. Abachi et al., Phys. Rev. Lett. 743548 (1995).

[2] M. Cacciari and P. Nason, UPRF-2002-4.

[3] D. Acosta et al.: Phys. Rev. Lett. 91241804 (2003), Phys. Rev. D71 032001 (2005);

V. Abazov et al.: Phys. Rev. Lett. 94161801 (2005), Phys. Rev. Lett. 94091802 (2005).

[4] Z. Nagy: Phys. Rev. Lett. 88122003 (2002), Phys. Rev. 068094002 (2003).

[5] S. Frixone and B. R. Webber: JHEP 0206029 (2002), JHEP 03080072003.

[6] J. M. Butterworth et al., Z. Phys. C72 6371996. 